\title{
Gold Electroforms and Heavy Electrodeposits
}

\author{
DECORATIVE AND INDUSTRIAL APPLICATIONS
}

\author{
Dirk Withey
}

Johnson Matthey Chemicals, Royston, Hertfordshire, United Kingdom

\begin{abstract}
Although electroforming in gold to form free-standing objects has been carried out for a number of years for decorative applications, it is only in recent times that improvements in bathcompositions and performance reliability bave made the use of the technology a practical proposition in selected high-technology applications.
\end{abstract}

The most widely accepted definition of electroforming is $\therefore$. the production of articles by electrodeposition where the final object is removed from the master or matrix on to which it is deposited.' (1)

Since, in most cases, the electroform must be selfsupporting, a primary requirement is that the final product must be strong and free from cracks and laminations caused by stress. The technique as applied to gold has not reached its full potential in either decorative or industrial fields because of difficulties with the early electrolytes which produced stressed, porous and badly distributed deposits. However, recent advances in gold plating technology have enabled satisfactory deposits with thicknesses of up to $6.25 \mathrm{~mm}$ to be achieved. Thus modern gold electroplating processes are capable of producing very fine-grained structures which are very much harder than those obtained by rolling or casting metal of the same purity. A wide range of articles can therefore now be electroformed. Provided that the surface of the initial matrix is capable of being made electrically conductive, a heavy coating of gold can be built up on virtually any object. Moreover, by using electroforming, large savings of metal can be made by reducing crosssection without sacrificing the required rigidity of the final product.

\section{Decorative Applications}

Flat objects - articles of this type are probably the easiest to produce by electroforming since any inconsistencies in the gold layer are hidden from sight when the object is mounted on a base. The most common items in this category are plaques, seals and medals.

In the first stage of the operation the artefact to be reproduced is used to produce a silicone rubber or P.V.C. negative using conventional moulding techniques. These materials give an excellent transfer of detail from the original and are sufficiently flexible to facilitate easy removal of the electroform at the completion of the process. Epoxy or acrylic compounds are used when a more rigid construction is required.

One great advantage with these moulding materials is that they can be used several times in the forming operation before requiring replacement so that manufacturing costs are substantially reduced.
Cylindrical shapes - as in the case of the production of the Prince of Wales coronet (2), these objects can be made by forming onto the inside of an open-structured cylindrical mould. A rigid, two-part construction is used to avoid flexing during deposition and to allow the final article to be removed at the end of the process.

Cylinders of gold can also be formed by electrodeposition on the outside of copper tube or wire. After forming, the copper is removed by dissolution in nitric acid. Gold tube fabricated in this way, although being almost 24 carat, is very much harder than that produced by conventional methods. The electroformed material can be cut into sections and machined to form rings which have a very high gold content and also excellent wear resistance.

Three-dimensional items - three-dimensional electroforms can be produced hollow and therefore enable substantially less gold to be used than when similar articles are cast by the lost-wax process. Thus as indicated earlier the additional strength imparted by the electrodeposition process means than an object with the same rigidity as a cast article can be produced, in many cases, with as little as 30 per cent of the gold required by the casting technique

A wax model, similar to that used in the lost-wax process, is produced from a rubber mould taken from the original. The surface is made electrically conductive and forming is carried out to the required thickness. The wax is removed in hot water to produce an electroform which rarely requires any subsequent chasing, polishing or other finishing. Very complicated shapes can be readily prepared without the need for final assembly of component sections.

Low melting point Cerro metals (tin-bismuth or leadantimony alloys) are useful alternatives to waxes but must be chosen carefully to avoid contraction during casting. These do not need to be metallized, as they are already conductive. They can also be recycled after they have been recovered from the electroform by heating in a silicone oil bath.

Natural objects - the true versatility of electroforming is probably best shown in its application to the production of replicas of natural objects. In most cases the forming process encapsulates the initial article which is allowed to decay inside. Items of jewellery can be produced from leaves, ferns, flowers 
such as orchids, sea-horses and many other naturally-occurring materials. Gold coral can be made by electroforming, the original being removed in dilute hydrochloric acid.

A difficulty that has to be borne in mind is that the porous nature of many natural objects makes them difficult to electroform and staining can occur as a result of the entrapment of electrolyte. This problem is greatly reduced by freeze-drying the article and coating its surface with a thin layer of lacquer or varnish before immersing it in the electroforming bath.

\section{The Metallizing Process}

Before electroforming can be carried out on non-metallic material such as waxes, rubbers and plastics, the surface must be made electrically conductive.

The original methods, involving the use of graphite or metallic powders, have been superseded in recent times by silver spraying and metal sputtering techniques. The application of a thin silver layer, as used in the production of mirrors, is probably the most popular method used by electroformers today (3).

An ammoniacal silver nitrate solution is sprayed through a twin-jet gun so that it mixes intimately with a reducing solution at the surface of the article to be metallized. A number of reducing agents are used, including dextrose, formaldehyde and hyrazine. Although the process can be hazardous due to the formation of explosive silver fulminate, it has the advantage of producing a bright, coherent, conductive silver layer. The thickness of the metallized layer is normally kept to about $0.1 \mu \mathrm{m}$ to avoid the build-up of stress in the deposit.

The coating is allowed to dry and a contact screw or pin is inserted and cemented with the use of a silver-epoxy metallizing preparation.

\section{The Electroforming Process}

The choice of electrolyte for the gold electroforming processing is of paramount importance. By far the greatest cause of problems in the past has been the use of baths which are unsuitable for heavy deposition. Many experimenters have tried to use electrolytes which were not specifically designed for electroforming and have produced stressed, cracked, nodular, rough deposits which were badly distributed or which reduced the amount of detail on the final surface.

The majority of these pitfalls can be avoided by the selection of the correct bath and operating conditions, and to this end an electrolyte based on the sulphite complex is normally used to produce a gold deposit with sufficiently low internal stress such that it will withstand the stamping operation necessary for hallmarking. Excellent metal distribution can be achieved when depositing from these baths and fine detail is not lost during the electroforming of three-dimensional articles.

The forming process is carried out by immersing the metallized master in the solution which normally contains gold

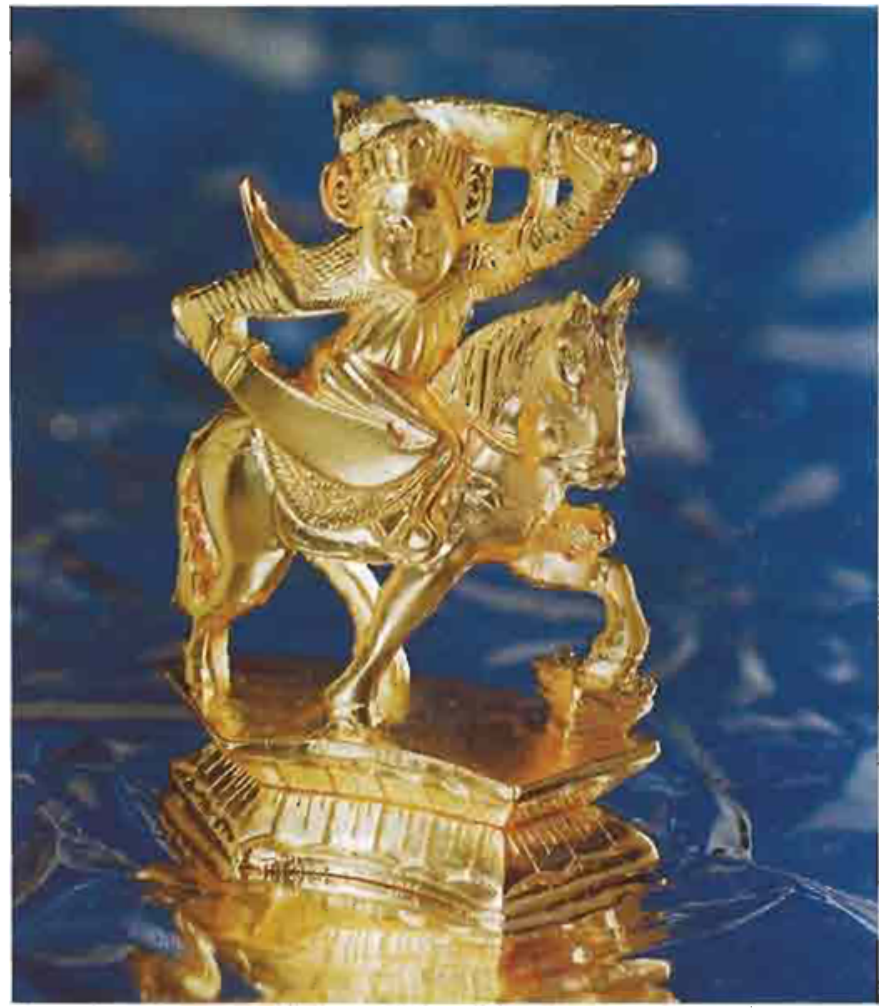

Chesspiece with a wall thickness of about $\mathbf{3 7 5}$ micrometres made from a solid wax model

at a concentration of 30 to $40 \mathrm{~g} / \mathrm{l}$. Initially a low plating current density is applied to produce a very fine grained structure and the current is gradually increased to around $0.5 \mathrm{~A} / \mathrm{dm}^{2}$ for the remainder of the deposition time. By this procedure, the formation of large crystals in the deposit is avoided and the risk of rough and nodular deposits is reduced. The electrolyte is constantly filtered through a fine polypropylene mesh cartridge and regular gold salt additions are made to maintain a stable metal content in the bath.

The forming process usually takes several hours to complete, after which the electroform may be removed and separated from the master to give a near-perfect reproduction of the original. Apart from trimming off rough edges and the possible removal of residual silver from the metallizing process, very little mechanical treatment is normally necessary.

\section{Industrial Applications \\ Electron Microscope Grids}

A major industrial application for gold electroforming is in the production of electron microscope grids by Veco Research B.V. (4). A pattern of the grid is produced on a nickel or stainless steel plate using a photoresist. Following a developing and baking process, gold is deposited on the unmasked areas. When 
the required thickness has been achieved, the metal plate is removed from the electrolyte and the electroformed grids carefully separated from the substrate.

Aperture diaphragms for electron microscopy are also produced by electroforming, as are special purpose electrodes for cathode ray tubes.

\section{Gold Coated Windscreens}

An airline pilot or railway driver requires a clear view ahead at all times. To help prevent icing and misting of windscreens, a transparent, electrically conductive, gold film is applied by sputtering from a cathode surface having a thick gold electrodeposit to the inner surface of the outer glass before laminating. This enables an electric current to be passed through the laminated sheet to maintain it at an elevated temperature without the generation of adverse optical effects, which are common with the alternative wire element systems.

The gold film windscreen has the double advantage of both reflecting U.V. radiation and absorbing some visible radiation. Not only is glare reduced in strong sunlight therefore but also, in hot weather, the amount of solar heat transmission is considerably reduced thereby ensuring a more comfortable temperature in the driving compartment. The improvement in working conditions resulting from the use of gold in their laminated, missile impact resistant, locomotive windscreens has led to the widespread adoption of such systems by the Italian, Swiss and Canadian Railways.

Similar systems have been specified for aircraft flight deck transparencies for a number of years, dating back to the de Havilland Comet which was the world's first jet airliner. More recently, the familiar pink tinge imparted to windscreens by gold sputtering has been evident on V-bombers and on Vickers V.C. 10, Trident and British Aerospace/ Aérospaciale Concorde aircraft.

\section{The Sputtering Process}

In the process used by the Aircraft and Special Products Division of Triplex Safety Glass, Birmingham, United Kingdom, a thin layer of bismuth oxide $\left(\mathrm{Bi}_{2} \mathrm{O}_{3}\right)$ is applied (5) before depositing the gold.

The gold film is then sputtered at a pressure of less than 12.8 $\mathrm{Pa}$ and with an applied potential of around $1 \mathrm{kV}$. The process is carried out in an argon atmosphere and uses a large cathode consisting of a steel-backed copper sheet on to which has been applied a gold coating usually about $500 \mu \mathrm{m}$ thick.

The resultant gold film is normally about $10 \mathrm{~nm}$ thick and it is given a further treatment to deposit a second layer of bismuth oxide to complete the $\mathrm{Bi}_{2} \mathrm{O}_{3} / \mathrm{Au} / \mathrm{Bi}_{2} \mathrm{O}_{3}$ sandwich. The metal oxide substrate enhances the conductivity and visual transparency of the layer, which is heated to $200^{\circ} \mathrm{C}$ for added stability. The strong binding force produced in this way prevents migration of the metal atoms and the formation of large grains.

\section{The Electrodeposited Gold Cathode}

The gold cathode used in the sputtering process is prepared by electrodeposition, the precious metal being applied in this way to provide the very fine grained structure and uniform metal distribution which greatly aids the subsequent production of a satisfactory gold coating on the glass.

After the initial cleaning processes, the copper-clad steel backing is immersed in the electrolyte and the deposition commenced. The application of a special paint to the sides and top of the cathode prevents the build-up of gold on these surfacess. The plating current is applied over several days to give a slow deposition rate and prevent the formation of a coarse structure. During the whole of this period the solution is constantly filtered, the cathode is rocked to disperse gas bubbles and regular additions of gold salt are made to maintain a stable metal content in the electrolyte.

At the end of the process the surface is cleaned and dried, and the masking paint removed. By this time the cathode will have increased in weight by around $8 \mathrm{~kg}$ of gold and will be ready for approximately another year in service before it next requires refurbishing.

\section{The Micro-electronics Industry}

Widespread use is made of gold in the electronics industry for sputtering or evaporation on to semiconductors. Sputtering targets normally consist of a disc of copper $25 \mathrm{~cm}$ in diameter and $0.5 \mathrm{~cm}$ thick. The surface is polished on one side and selectively plated to form a pure, fine-grained deposit up to $200 \mu \mathrm{m}$ thick.

Very pure gold is required, particularly in the case of the production of Random Access Memories (RAM's), where the occurrence of 'soft' errors is normally due to the presence of uranium or thorium at ppb levels of concentration. When an electrolytic method is used for the production of the gold targets, carry-over of impurities can be reduced and hence the level of the emission of alpha-particles minimized.

Recent measurements by autoradiography, carried out by the Atomic Energy Research Establishment, Harwell, show that it is possible to reduce the incidence of alpha-particles to $0.0001 / \mathrm{cm}^{2} / \mathrm{h}$ when using high purity deposits from a nitritosulphito gold electrolyte.

\section{Electron Microscopy}

A recent article (6) demonstrates how the application of deposits of gold improves the contrast and detail of Scanning Electron Microscopy samples.

The cathode material in the sputtering process is a small copper disc, electroplated on one side with gold to a thickness of several hundred micrometres. The electrode is bombarded with 
Italian State Rail D445 locomotive with a gold coated windscreen giving reduced glare and reduced solar heat transmission through the glass

positive ions of argon at a potential of around $2 \mathrm{kV}$ and under reduced pressure. The gold is sputtered off the target and redeposited randomly on to the sample, giving excellent distribution even in deeply recessed areas. When the gold is expended, the cathode is removed and can be readily refurbished with a new coating.

This application contrasts interestingly with the use of transparent gold films, $30 \mathrm{~nm}$ thick, in Transmission Electron Microscopy in the study of defects, intermetallics and analysis of stresses in films, described elsewhere in this issue.

\section{The Nuclear Industry}

\section{Nuclear Fuel Reprocessing}

Magnetic separation has been developed by British Nuclear Fuels (7) as a process for the removal of unwanted fission products from highly irradiated nuclear fuels, such as those arising from reactors using uranium oxide.

When the fuel is dissolved in nitric acid several of the fission products remain insoluble. It is desirable to remove these residues before proceeding with the subsequent solvent extraction process and high gradient magnetic separation has been shown to have a number of advantages over conventional methods for application in a radioactive plant (8).

In the filtration process a matrix of $3 \mathrm{~mm}$ diameter balls is subjected to a magnetic field of between 0.8 and 1.7 Tesla and the nitric acid, containing the reactor products, is passed through at a flow velocity of between 0.6 and $1.6 \mathrm{~cm} / \mathrm{s}$. A large proportion of the unwanted material is magnetic and is trapped on the surface of the balls. When the filtration is completed up to 98 per cent of the suspended solids are removed with up to 7 per cent of its own weight being deposited on the filter. The solids are readily flushed from the balls by removing the magnetic field.

The normal construction materials for nuclear plant are austenitic stainless steels. These show very low rates of corrosion in nitric acid, but, unfortunately, are not ferromagnetic. In order to obtain a suitable matrix of balls for the process, a ferromagnetic steel is used, but, to enable the balls to withstand the corrosive environment, they are given a thick electrodeposited coating of gold. Since the steel balls have to withstand boiling $3 \mathrm{M}$ nitric acid, a minimum deposit thickness of $37.5 \mu \mathrm{m}$ is specified. Spheres coated in this way show no signs of attack or weight loss over periods in excess of $5000 \mathrm{~h}$.

Gold cathode being washed following the deposition of around $8 \mathrm{~kg}$ of the metal
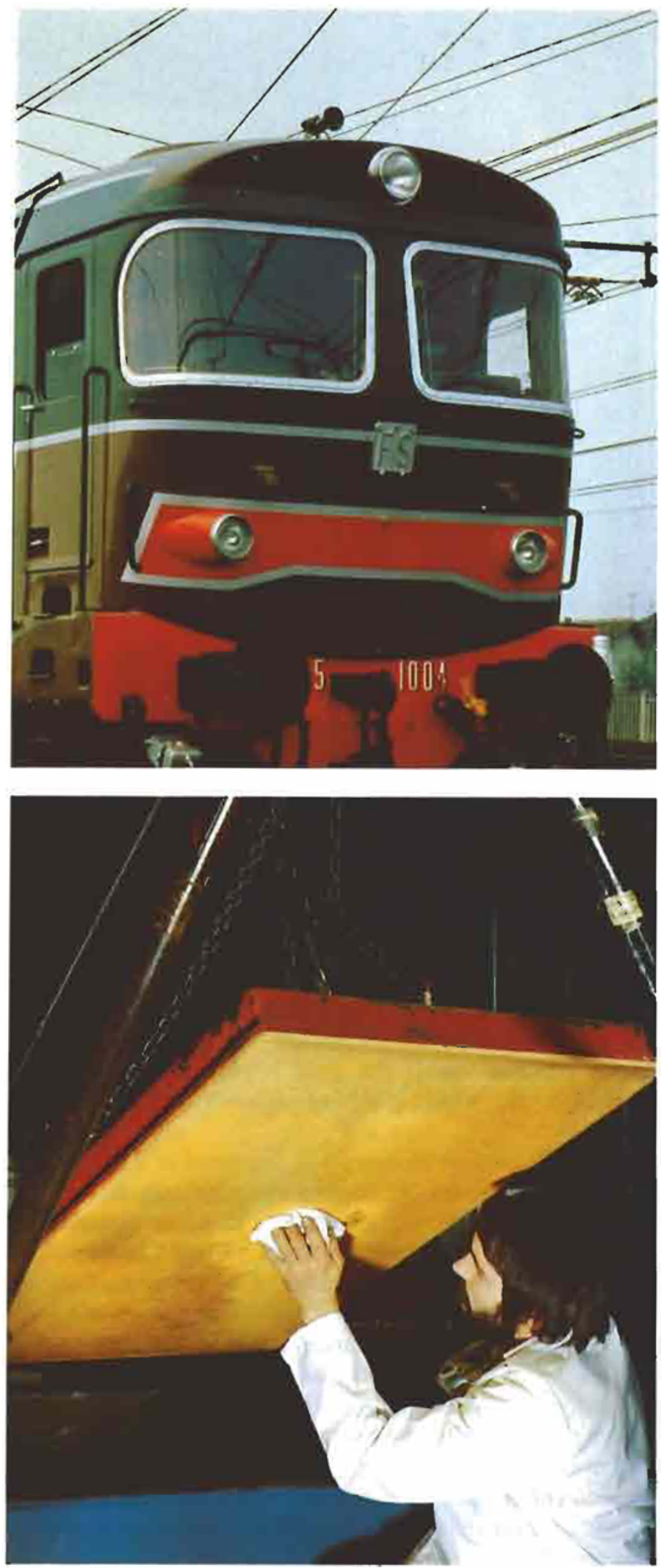


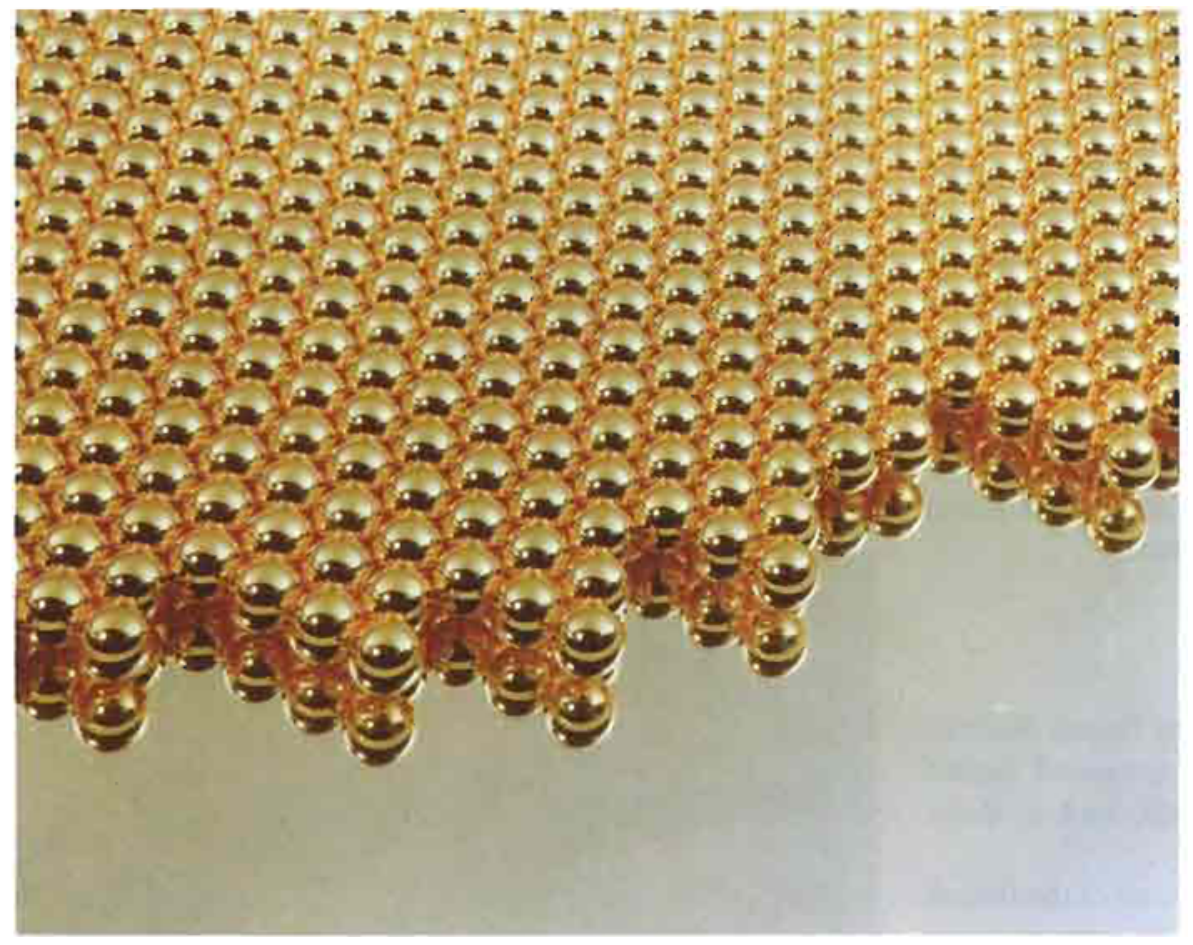

Steel balls, used in nuclear fuel reprocessing, electroplated with $\mathbf{4 0}$ micrometres of low-porosity gold to give a corrosion-resistant coating in nitric acid having an estimated service life of 30 years

The presence of the gold does slightly reduce the efficiency of the magnetic collection but the performance of the filter is not significantly altered.

Other future applications of the method include its possible use for the filtration of chromium plating baths and other strongly acidic solutions.

\section{The Gold Plating Process}

To provide the maximum possible protection without using excess precious metal, the gold is plated from a special electrolyte (9) which produces deposits having very low porosity.

The balls are first ultrasonically degreased in a chlorinated solvent and then further cleaned electrolytically in a mildly alkaline bath. Following a treatment in dilute hydrochloric acid, the spheres are given an initial coating of nickel. The components are plated in batches of 10000 using a nickel sulphamate solution which gives a low-stress deposit and good compatibility with the substrate material. The operation is carried out in an immersed plating barrel equipped with a polypropylene mesh lining which allows good solution replenishment within the cylinder.

A thin coating of copper is next applied to promote good adhesion of the final gold layer which, at a thickness of around $40 \mu \mathrm{m}$, has been estimated to be sufficient to give a service life of 30 years.

\section{Isotope Production}

The Cyclotron Unit of the United Kingdom Medical Research Council produces a wide range of isotopes which are used extensively in the detection of diseases. Many of these materials lose their activities very quickly and recently the supersonic aircraft Concorde has been employed to help deliver a krypton isotope to hospitals in the U.S.A. within hours of its manufacture.

In the production process, a target material is bombarded by a stream of high-energy sub-atomic particles which are accelerated in the cyclotron to a velocity of around $32000 \mathrm{~km} / \mathrm{s}$.

To focus the beam centrally and to enable the equipment to be effectively closed down, a series of shutters is used. These are fabricated in copper and are electroplated with $500 \mu \mathrm{m}$ of gold to provide a surface which is capable of absorbing the high energy beam and leaving little residual activity. The gold coating also provides excellent thermal conductivity and enables efficient heat dissipation to be achieved.

As well as producing isotopes which can be used for diagnosis, the Cyclotron Unit is also able to provide treatment for certain tumours by neutron irradiation. A beam of deuterium particles is guided by a gold-plated iris assembly on to a beryllium target which then emits the neutrons required for the treatment of the patient. The gold is again used to give good absorption of the radiation and, by the measurement of temperature variations across the iris assembly, accurate alignment of the beam is made possible. 
Shutter assemblies from the Cyclotron Unit of the Medical Research Council at Hammersmith Hospital, London with a surface of electrodeposited gold of about 500 micrometres thickness which absorbs high energy particles leaving little residual activity

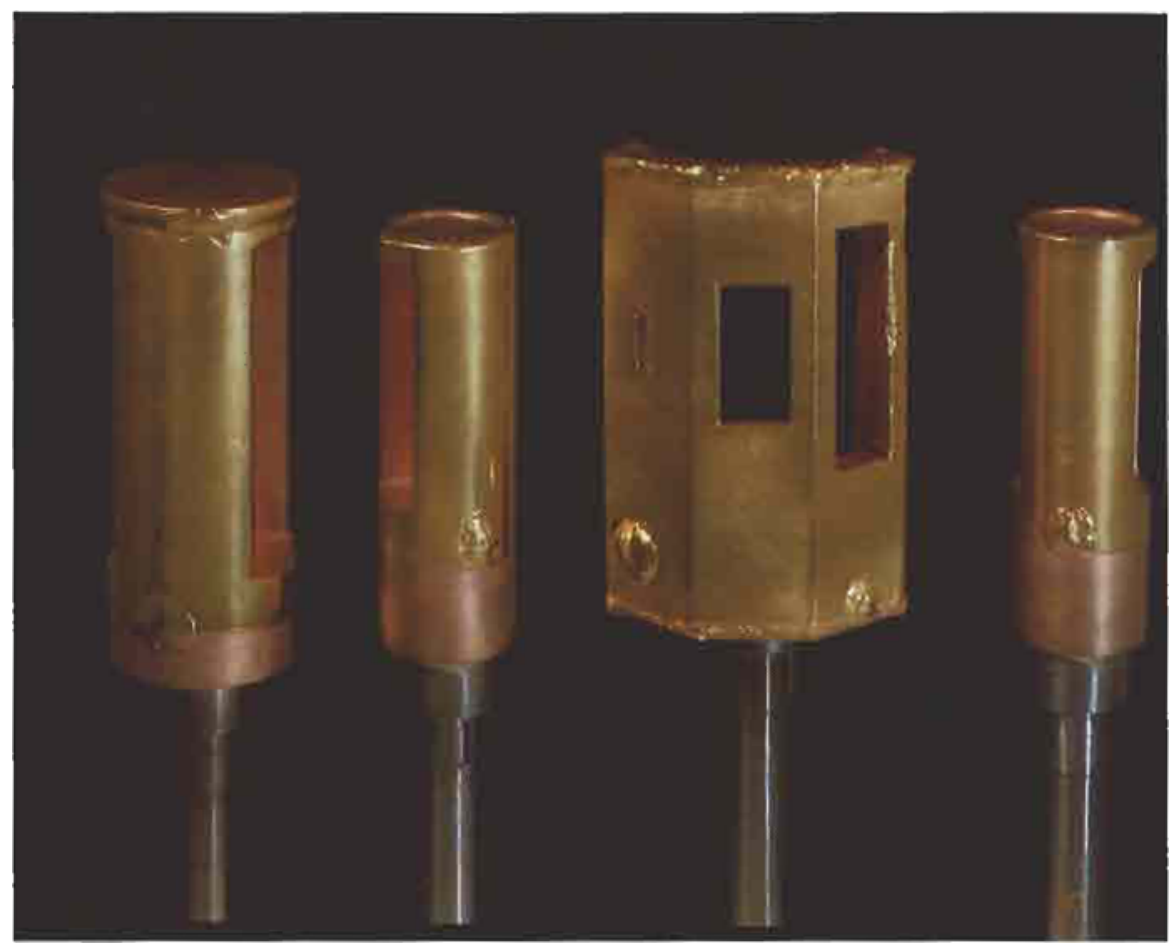

\section{Conclusions}

The uses of heavy gold deposits and electroforms in both the industrial and decorative fields are many and varied.

Now that the initial difficulties in finding suitable electrolytes have been overcome, thicknesses of several hundred micrometres can be obtained. This, coupled with the use of modern techniques such as ultrasonics and pulse-plating which modify the deposit growth and structure, should ensure that many more applications for heavy electrodeposits of gold will be found in the future.

\section{References}

1 P. Spiro, 'Electroforming', Robert Draper Ltd., Teddington, 1971

2 D. R. Mason, 'Gold Plating Technology', Electrochemical Publication Ltd, , Ayr, 1974

3 R. W. Furness, 'The Practice of Plating on Plastics', Robert Draper Ltd., Teddington, 1966

4 A. Mohan, Gold Bull., 1975, 8, (3), 66-69

5 L. Holland, 'Vacuum Deposition on Thin Films', Chapman and Hall, London, 1956

6 J. T. Fourie, Gold Bull., 1982, 15, (1), 2-6

7 U.K. Pat. 1 555670 , Nov. 1979

8 J. A. Williams et al. 'The Application of High Gradient Magnetic Separators in Nuclear Fuel Reprocessing', Nucl. Technol., 1982, 52, (2) 284.204

9 P. C. Hydes and H. Middleton, Gold Bull., 1979, 12, (3), 90-95 